\title{
CONFERÊNCIA
}

\section{INFANCIAS Y CULTURA EN LA ACTUALIDAD LATINOAMERICANA. CÓMO ATENUAR LAS INFLUENCIAS EXÓGENAS}

\author{
Elbia Haydée Difabio ${ }^{1}$
}

\section{PRIMER MOMENTO: INFANCIAS, CULTURA E IDIOSINCRASIA}

Conviene comenzar precisando los conceptos de la mano del Diccionario de la Lengua Española. Según él, se define infancia como el período de la vida humana desde el nacimiento hasta la pubertad, o sea, hasta la primera fase de la adolescencia. Ahora bien, se prefiere pluralizar el término para dar cuenta de la diversidad que contiene esa franja etaria, de las diferentes dimensiones y de sus muchas complejidades. Por su parte, se adopta la tercera acepción de cultura2: "Conjunto de modos de vida y costumbres, conocimientos y grado de desarrollo artístico, científico, industrial, en una época, grupo social, etc.". Y, en cuanto a "idiosincrasia", su semántica abarca los rasgos, el temperamento, el carácter, distintivos y propios de un individuo o de una colectividad.

\footnotetext{
1 Profesora, Licenciada y Doctora en Letras. Categoría 1 en SIIP. Dirige y codirige becarios y tesistas de grado y de posgrado en UNCUyo, UCC y UNSJ. Colabora en Banco de Consultores de CONICET, Registro de Expertos de CONEAU, Banco de Evaluadores de la Secretaría de Investigación y Desarrollo de UBA, UNTREF, UNQ, UM, UNSJ, UNLPam, UNT, UNHUR, UCA y UCCUyo. Autora y coautora de libros, evaluadora (en el país, México y Chile) y autora de artículos en revistas especializadas y Actas en Argentina y en el extranjero (España, Chile, Venezuela, Bolivia, Brasil). Miembro de Comités Científicos Internacionales en Argentina, México, Chile y España. Orcid iD: http://orcid.org/0000-0003-2695-2299. E-mail: elbiad@ffyl.uncu.edu.ar

2 Infancia, cultura, pubertad y adolescencia proceden todas del latín: respectivamente,

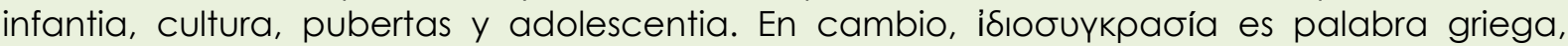
formada por el adjetivo î́ı

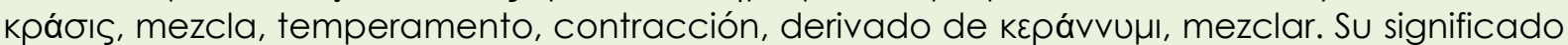
original es "temperamento particular' y en forma extensiva, se aplica a los conglomerados humanos -pueblos, razas, sociedades.
} 


\section{SEGUNDO MOMENTO: SITUACIÓN PRESENTE}

Asumiendo como bases las nociones anteriores, es indudable que las costumbres de nuestros niños se están transformando vertiginosamente. Los factores son múltiples, decisivos y complementarios: adultos ausentes también por diversas razones-; la mayoría de las escuelas anacrónicas o distraídas ante esta realidad que las desborda; métodos pedagógicos clonados del extranjero sin adaptación a las aulas, un sistema educativo fragmentado; sobre todo en las urbes, inseguridad para salir solos, pocos espacios disponibles al aire libre y viviendas reducidas, paseos "obligados" a centros de compra globalizados como los shoppings y los malls; el consumismo vigente, la propaganda comercial, tendencias cambiantes de modo acelerado por un mercado exógeno avasallador que impone modas y gustos en vestimenta, comida, diversiones, juegos y deportes; literatura, sus personajes y héroes, modificaciones en la sensibilidad estética, en los comportamientos, posturas y expresiones verbales; la dispersión, las nuevas formas de encarar la amistad y las relaciones sociales; situaciones de riesgo y de violencia, entre ellas el acoso cibernético; la niñez con multitarea simultánea y/o hiperconectada... Así, por ejemplo, en muchos lugares se celebra Halloween, a manera de copia y sin análisis del significado de esta tradición originariamente celta; se consumen videos juegos en línea como Among Us sin ninguna criba; incluso se inculca la obligatoriedad de estudiar inglés por sobre todos los demás idiomas, en tanto vehículo universalizado de comunicación y sin espacio para otros.

A propósito del inglés, Internet -por lo demás, medio de comunicación prioritariamente comercial- enumera los juegos más esperados en 2020 y detalla: Cyberpunk 2077, Dragon Ball Z: Kakarot, Guilty Gear Strive, Final Fantasy VII Remake, Nioh 2, Wasteland 3, Vampire: Bloodlines 2, Elden Rings. Ni siquiera se traducen los títulos. Los niños los conocen desde el principio en su idioma original. En YouTube la más popular es la serie de videojuegos de Minecraft. Y podríamos continuar con los canales televisivos para pequeños: es la misma primacía, aunque con doblaje. 
Estas novedades -que además proliferan sin descanso y son imparables, frenéticas y abruptas-, se posicionan con bloques para armar, disfraces, calcomanías, libros para pintar, camisetas, gorros, viseras y sombreros, muñecos y peluches -en variados materiales, incluso tejidos-; platos, vasos y tazas; golosinas -como huevitos sorpresa-, útiles escolares, lámparas, pijamas, toalla y toallones, ojotas y zapatillas... No queda un rincón libre sin ocupar. La parafernalia es, como su mismo nombre lo indica ${ }^{3}$, un alarde de exhibición y de adoctrinamiento que somete a una masiva elección. Y mutan de modo rápido: ya han perdido cierta vigencia los Minions, esas criaturas amarillas que surgieron en el 2015. Los pitufos van renovándose: de serie televisiva de 1981 a 1990, devino película, la primera, en 2011, aunque fueron creados por el dibujante belga Peyo en 1958.

En cambio, y lamentablemente, Los Peques, serie de televisión de animación en 3D creada en Argentina que narra las vivencias de unos duendes en la Patagonia argentina, no alcanzó más de 23 episodios. Apareció en 2001 en la provincia del Neuquén se difundió por Cable Visión del Comahue. Hoy los niños de mi país conocen a Zamba. Este personaje de la cadena de televisión infantil Pakapaka perteneciente al Sistema Federal de Medios y Contenidos Público suma siete temporadas con 58 episodios de 20 '. Sin embargo, ignoro si es conocido en algún otro país de Latinoamérica.

También los "superhéroes" son foráneos: Superman, Spiderman U Hombre Araña, Hulk, Deadpool, Ajax, Angel Dust y tantos más. Lejos están del código heroico ejemplar, ya que los pueblos primeros hicieron remontar hasta ellos su origen, haciéndolos protagonistas de su protohistoria y exponentes de su espíritu. Se caracterizaban por tener conciencia, por un lado, de su grandeza y, por otra, de sus limitaciones como humanos. "La figura del héroe, como la del santo, más que mitos son arquetipos culturales

\footnotetext{
3 Parafernalia: Del inglés paraphernalia, y este del latín medieval paraphernalia 0 parafernalia [bona] '[bienes] parafernales'< parafernales. Del latín medieval parafernalis, derivado a su vez del latín paraferna 'dote', y este del griego mapápqpva: bienes que trae una novia como dote.
} 
heredados de un pasado remoto en los que la realidad histórica y los embellecimientos de la imaginación se han imbricado inextricablemente"4.

Es un ideal humano, como el santo y el sabio, y conlleva por ende valores, todos en torno de lo espiritualmente noble, que podríamos equiparar con las virtudes cardinales de prudencia, justicia, fortaleza y templanza. Alcanzarlas y mantenerlas es una búsqueda personal, ardua, continua, comprometida e intransferible. Cada héroe se hace héroe con el otro, no a espaldas o a expensas del otro. El fin primordial es vencerse a sí mismo. No se sustrae del sufrimiento y del pesar. Aunque puede acceder a los Campos Elisios, está privado de inmortalidad (con excepción de Heracles, el único

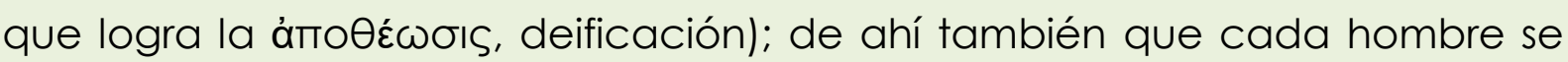
sienta próximo al héroe en general o a uno en particular.

En definitiva, los héroes resultan formas cardinales de ejemplaridad vital; modelos de comportamiento, paradigmas altamente provechosos, educadores natos. Guían para la libertad, la autodeterminación, la interactuación. Nos muestran el camino, nos hacen partícipes de su experiencia.

Valga esta digresión para cotejar con los pseudo-héroes vigentes: ¿̇Cuánto comparten del ADN de los primigenios paradigmas? ¿̇Por qué no se difunden los héroes de cada país latinoamericano entre nuestros países? Seguramente, falta de presupuesto, pero también de iniciativa gubernamental.

\section{TERCER MOMENTO: HACIA LA RECUPERACIÓN DE NUESTRA IDIOSINCRASIA}

Urge reivindicar las diversas procedencias fundantes de la identidad cultural de América Latina: la autóctona, esto es, de los pueblos originarios; la ibérica y subsahariana en su origen, y el resto europeo (tanto latino como de los ámbitos germánico, céltico, eslavo y anglosajón) junto con influjos del mundo árabe (en especial el Levante) y del Asia Oriental. ¿Cómo llevar a cabo el intento de dicho rescate?

\footnotetext{
4 Bauzá, H. F. (1998: XI). Conceptos del prologuista español Luis Gil.
} 
El neurólogo argentino Facundo Manes, creador del Instituto de Neurología Cognitiva, ha estudiado cómo incide en las mentes infantiles el tiempo de pantalla y la Organización Mundial de la Salud recomienda prohibir el uso excesivo de estos dispositivos y aplicaciones a los menores de 2 años y a partir de esa edad, el límite no debe superar de 1 a 2 horas al día y supervisando la calidad de los contenidos. Si se respetan estos parámetros, mermaría buena parte de los riesgos enumerados sobre la desmedida globalización que perjudica la cultura propia de cada país ${ }^{5}$. También la psicóloga clínica Elizabeth Kilbey advierte sobre los peligros del abuso digital y/o del empleo indiscriminado en la etapa que ella denomina de "latencia", "que cubre el período que va de los 4 a los 11 años más o menos, es una de las etapas más descuidadas, pero más importantes, en el desarrollo del niño" (2018, s.p.). Por su parte el DLE registra el término como "Tiempo que transcurre entre un estímulo y la respuesta que produce". Estos años representan una fase fundamental en el desarrollo del cerebro caracterizado por una sorprendente plasticidad neuronal, que se ve perjudicada a causa del exceso de pantalla.

Una segunda solución es orientarlos a la observación de su entorno, de su realidad, a estimular el disfrute y la interacción, la convivencia, con ella, a explorarla, interpretarla, apreciarla, admirarla, respetarla y protegerla, a ocuparse y a convivir con su ambiente; a identificar sus elementos y a jerarquizarlos, aprendiendo también a sopesar posibles riesgos y peligros a fin de prevenirlos. Si el niño se solidariza con su entorno, se estará formando en la ecología. Si encaminamos la tarea a historias locales, a la naturaleza inmediata y cercana, paliaremos el avance constante de prácticas culturales ajenas -a veces, inclusive contrarias- al sentir de la comunidad. Estaremos cimentando raíces. No se excluye de ninguna manera la fantasía, que resulta la elaboración alternativa de las relaciones con los contextos

\footnotetext{
5 A su vez, explica Swing en Kilbey (2018, p. 192): "Un estudio constató que los niños que exceden las 2 horas recomendadas de tiempo diario ante una pantalla digital tienen una probabilidad de 1,5 a 2 veces mayor de sufrir problemas de atención en clase". La investigación mostró asimismo que esos niños presentaban menor capacidad de autocontrol y tendían a ser más impulsivos.
} 
cotidianos. Sí se hace hincapié en la presencia deliberada y sistemática de la realidad, factible para cualquier adulto que quiera ayudar a los infantes. Además, la presencia del adulto mediador da sentido a los aprendizajes; no una pantalla que carece de filtro, que no procesa ni mide la información emitida. Así, la labor se humaniza.

Contemplar la realidad implica detenerse en el espacio circundante, en sus sonidos y aromas, en su movimiento y color, en sus luces y sombras, en sus sabores y cualidades hápticas ${ }^{6}$. A partir de las recientes investigaciones de la pedagoga canadiense Catherine L' Ecuyer $(2015,2017)$ se ha constatado que el aprendizaje infantil se alcanza mediante experiencias sensoriales concretas para comprender el mundo y para comprenderse a sí mismos. Efectivamente, los últimos estudios en neurociencia corroboran que la memoria semántica (de conocimientos conceptuales) y la biográfica (de los acontecimientos vividos mediante experiencias percibidas) aún no están diferenciadas en la infancia. Las dos formas de esta facultad psíquica se separarán gradualmente en el transcurso de la adolescencia hasta la adultez, lo que prueba que los niños no aprenden a través de discursos, fichas o pantallas, sino que necesitan experiencias reales y relaciones interpersonales "en directo". Este acercamiento a un animal, a una fruta o a una flor "verdadera", a un amiguito o compañero de juegos, a una salida al aire libre, lo irá distanciando de las "ventanas" digitales del mundo y podrá, por ende, impregnarse del amor por su terruño y por sus costumbres. Si no podemos armar una huerta escolar, visitemos una familiar. Cultivemos flores y cuidarán más las que encuentren por el camino. Organicemos bandas y coros infantiles. Incursionemos en la cocina con platos típicos sencillos, ayudados por los adultos.

Ahora bien, la motivación debe ser interna $y$, sobre todo, trascendente; o sea, según un ideal de bien, verdad y belleza, en función de los fines. Junto con entroncarse con la realidad -offline, no online-,

\footnotetext{
6 Una educadora belga, Hélène Lubienska de Lenval, discípula y colaboradora de María Montessori y posteriormente de Pierre Faure, toma como medios de sus prácticas educativas el silencio, el rito, la contemplación y el trabajo personal. Si bien muchos de sus consejos se orientan a la catequesis, es factible extrapolarlos a la laicidad. Vale la pena leerla.
} 
corresponde hermanar a los niños con la humanidad. Es más, ellos mismos pueden ofrecer alternativas para ajustar las propuestas. Y para ello, también el adulto debe mantener intacta su capacidad de asombro, que en el niño es un mecanismo innato y de conexión con el entorno espacio-temporal, para compartir su contacto empático con la hermosura de un paisaje "en directo". A propósito del adulto, una copla de Alfredo Bufano (1983) canta:

\author{
Ante una flor de los campos \\ me pongo a considerar \\ que hay muchos hombres que nunca \\ han aprendido a mirar.
}

En este marco, hemos de recuperar la cultura del esfuerzo. Hoy ha devenido casi una mala palabra 0 , al menos, una palabra antipática, que conviene silenciar. Y el esfuerzo supone atención, voluntad, autocontrol, templanza, paciencia, constancia, responsabilidad, confianza, convicción, honestidad y resiliencia. Es apropiado traer a colación aquí un pasaje de Alejandro Dolina en "La aventura del conocimiento y el aprendizaje" (2011) que, si bien está referido a los adultos, podríamos muy bien trasladar a la etapa objeto de estudio porque, si no inculcamos desde temprano esta actitud de empeño, el resultado futuro será como bien describe el escritor y músico argentino:

Todos nosotros habremos conocido un número prodigioso de sujetos que quisieran ser ingenieros, pero no soportan las funciones trigonométricas. O que se mueren por tocar la guitarra, pero no están dispuestos a perder un segundo en el solfeo. O que le hubiera encantado leer a Dostoievski, pero les parecen muy extensos sus libros. Lo que en realidad quieren estos sujetos es disfrutar de los beneficios de cada una de esas actividades, sin pagar nada a cambio. Quieren el prestigio y la guita $^{7}$ que ganan los ingenieros, sin pasar por las fatigas del estudio. Quieren sorprender a sus amigos tocando "Desde el Alma" sin conocer la escala de si menor. Quieren darse aires de conocedores de literatura rusa sin haber abierto jamás un libro. Tales actitudes no deben ser alentadas.

\footnotetext{
7 Guita: Término argentino lunfardo por "dinero, moneda".
} 
Un aporte interesante para enseñar a filosofar desde el realismo surgió en la ciudad de San Luis, Argentina, en 2020 y continúa. Se trata de un programa único en su estilo sobre Filosofía realista en el Nivel Primario. Lo cursa personal directivo, asesor y docente de distintas provincias del país, con una convocatoria nutrida y entusiasta, que luego lo aplica en los colegios donde ejercen. Esta experiencia pedagógico-didáctica se implementa desde hace más de diez años en los colegios dependientes de la Universidad Católica de Cuyo-sede San Luis y su corolario fue la elaboración de una tesis de Posgrado pronta a ser publicada. La secuencia se inicia en primer grado de manera propedéutica, educando en la observación y en la admiración de la realidad. El ocio, el silencio, el asombro y el gozo conforman la vía para generar una actitud contemplativa de dicha realidad. En segundo grado, a partir de la Física aristotélica, se los dispone a descubrir la admiración como origen permanente y constante del filosofar y el asombro frente al cosmos, el orden, el movimiento $y$ especialmente la constitución de las cosas (materia y forma). En tercero se los introduce en temas propiamente metafísicos como substancia, accidente, acto, potencia, las cuatro causas, la causa última de todas las cosas. En cuarto grado se apela a la Antropología: el ser humano como persona, su naturaleza específica, las facultades humanas y su apertura a la trascendencia. En quinto el eje es la Ética: el fin del hombre, la felicidad, los actos humanos, moralidad del acto voluntario, las pasiones, los hábitos, las virtudes intelectuales y morales. En sexto grado se aplican lineamientos esenciales de la Lógica, mediante las tres operaciones: primera (concepto), segunda (juicio) y tercera (razonamiento).

En esta iniciativa académica soy responsable de un módulo que titulé "La literatura para niños como una puerta para el asombro". Me propongo desarrollar la capacidad crítica para seleccionar lecturas como complemento del programa, consciente de que la Literatura funciona como brújula y consejera, puente y aliada de la Filosofía; en este caso, un disparador entonces para el diálogo filosófico. Pero siempre el primer peldaño es la contemplación de la realidad. Y constantemente apoyado en 
el asombro, promotor de interés y puntapié inicial para el descubrimiento del mundo y para el conocimiento.

En tercer grado, por ejemplo, se estudia potencia y acto, sustancia y accidente, con sus tipos: cantidad, cualidad, relación, lugar, posición, posesión, tiempo, acción. Todo lo que existe tiene cuatro causas y podemos conocerlas preguntando qué, con qué, quién y para qué de las cosas. Así de llano y así de profundo... ¿ Qué mejor que observar el cielo durante unos días? Ahora bien, ¿cómo incorporar el arte literario? Por ejemplo, ofreciendo textos descriptivos. Las nubes han inspirado a muchos escritores, de los que se recuerdan algunas poesías:

$\begin{array}{lll}\text { Silveta } & \text { Se aleja } & \text { La nube } \\ \text { de lana } & \text { tan suave } & \text { remota } \\ \text { inmóvil, } & \text { con pico } & \text { apenas } \\ \text { lejana. } & \text { de ave. } & \text { se nota. }\end{array}$

Sosa, Amílcar Urbano (1963). Antología del Meñique. Mendoza: Ediciones El Pelícano, p. 13.

\begin{tabular}{|c|c|c|}
\hline $\begin{array}{l}\text { Platos voladores } \\
\text { y desconocidos } \\
\text { parecen las nubes } \\
\text { en su recorrido. } \\
\text { Como los linyeras, } \\
\text { gachos y en sigilo, } \\
\text { las nubes recorren } \\
\text { senderos dormidos. } \\
\text { Como promesantes, } \\
\text { despacio y de a una } \\
\text { navegan las nubes } \\
\text { siguiendo a la luna: } \\
\text { Va una nube densa }\end{array}$ & $\begin{array}{l}\text { llena de elefantes, } \\
\text { elefantes grises, } \\
\text { grises caminantes. } \\
\text { Y una muy oscura } \\
\text { de cabellos negros, } \\
\text { las crines al viento, } \\
\text { relinchos, resuellos. } \\
\text { Otra se dibuja } \\
\text { terrible y llameante, } \\
\text { con jaguares rojos } \\
\text { y relampagueantes. } \\
\text { Avanza esa blanca } \\
\text { de cisnes y garzas, }\end{array}$ & $\begin{array}{l}\text { cruza el firmamento } \\
\text { como una barcaza. } \\
\text { Cerrando el desfile } \\
\text { una nube rosa, } \\
\text { toda de sonidos } \\
\text { y de mariposa. } \\
\text { Larga caravana } \\
\text { de extraños } \\
\text { camellos } \\
\text { me gusta montarlos; } \\
\text { y explorar el cielo. }\end{array}$ \\
\hline
\end{tabular}

Ballarini, Olga (2009). Aprendiz de mago. Mendoza: Ediciones del Autor.

Bellos ejemplos, ¿̇verdad? Los dos son poetas de mi "patria chica", son mendocinos. Ahora bien, żnos hemos detenido con nuestros alumnos a observar primero el cielo y las cambiantes formas, tamaños, alturas y colores que las nubes adoptan? ¿Hemos explicado la diferencia entre una nube y un 
nubarrón?̨8 ¡Qué afortunados si se forma un arcoíris! Primero, la experiencia directa; luego, el arte: la palabra poética, la danza, una canción, modelado, títeres en que las nubes estén personificadas, cuadros o ilustraciones, dibujos propios...

En tanto los sentidos nos dan una primera información sobre el mundo, es fundamental que en la primera fase el diálogo también se oriente a la experiencia vivenciada. En estas clases, se intenta que adviertan que, en todo cambio accidental, una sustancia varía (y permanece) y algo se adquiere o se pierde durante el cambio. El sujeto o sustancia sigue siendo idéntico a sí mismo, pero alcanza un determinado accidente. Y se continúa con más profundidad: señalarles que puede haber cambios, en este caso que afectan intrínsecamente al componente principal de los cuerpos, son los cambios sustanciales. Para refutar algunos errores, es de suma importancia la distinción entre ambas clases de cambios, sustanciales y accidentales, para que sepan, al menos, que el crecimiento o desarrollo de un ser es un cambio meramente accidental. Algunas preguntas vinculadas con la observación de las nubes, que dan lugar a la búsqueda posterior de adivinanzas y de canciones y a la producción individual y/o colectiva de textos afines:

- ¿̇En qué fijamos la atención cuando mirábamos el cielo durante estos días?

- ¿̇Lo hacemos comúnmente? ¿Por qué no?

- ¿La prisa ayuda a advertir los detalles?

- ¿Por qué mirar con atención y con calma causa alegría?

- ¿̇Han ido cambiando las nubes? ¿QQué perdieron en el cambio?

- ¿Pueden trasladarse de un lugar a otro? ¿Por qué?

- ¿Con qué velocidad recorrían el firmamento? ¿Por qué?

- ¿̇on necesarias? ¿Por qué?

- ¿Qué permanece igual en ellas, a pesar de los cambios?

- ¿Serán suaves, serán frías, serán pesadas? (Invitación a investigar...)

8 Se aconseja para los más chicos el cuento rimado Doña Nube y Don Nubarrón de Pedro Villar. 
- ¿Qué les causó admiración?

- ¿Les ha pasado antes asombrarse por una nube diferente? ¿Cuándo?

- ¿̇Alguien ha atravesado una nube viajando en avión? ¿̇Qué sintió?

Es la alianza con lo pequeño, con lo cotidiano, enmarcado en un territorio determinado y sin premura, deleitándose con cada momento en serena secuencia: una piedrita jaspeada, una hoja multicolor, un charco casi inadvertido, un tronco con forma insólita, una rama danzarina, un gorjeo recién estrenado, la mirada tierna de un cachorro, las figuras proyectadas por la sombra de un árbol frondoso, la armonía de una escultura en la plaza cercana, el aroma del pan recién horneado en la panadería del barrio... Además, para identificar sonidos deberán ejercitar el silencio. Que se sensibilicen con la maravilla de su entorno y comprendan la vital vinculación que implica la convivencia entre el ser humano y la naturaleza animada e inanimada. Que presten atención a quien está al lado: a su compañero de mesa, a un anciano solitario o un placero a la espera de una palabra amable, a un perrito desorientado... Que tiendan al bien común. Que recuperen el aquí y el ahora.

\section{A MODO DE CIERRE}

El planteo explicado dista de ser una utopía. Aceptada la globalización imperante, podemos nutrir a la niñez de un sentido de identidad personal y comunitaria, mediante la frecuencia deliberada y sistemática emparentada con la realidad. La educación supone práctica, no solo debate. Apremia promover y diversificar experiencias y crear conscientemente acciones reparadoras, mediante prácticas comunitarias, pero con temas medulares por donde la vida transita. Habilitemos a la pregunta constante y propiciemos el acto creativo. No hacen falta recursos materiales, menos aún onerosos.

La escuela debe emancipar y habilitar espacios para velar por la memoria de una ciudad, de un país. Es, sin lugar a dudas, el agente 
fundamental; por ende, puede perforar los reclamos de la época, rebelarse ante esas exigencias que demandan que la niñez debe ser adulta lo antes posible. Los docentes no somos extras del sistema, todo lo contrario; de modo que busquemos esencialidad y especificidad por sobre los gobiernos educativos y las industrias mediáticas. Recompongamos la fragmentación, mostremos la satisfacción de los vínculos no utilitarios y volvamos a la centralidad y al cuidado de aprendizajes auténticamente significativos. En definitiva, favorezcamos la reflexión y focalicémonos en la cultura del aprendizaje colaborativo. Así y solo así protegeremos a la(s) infancia(s) y rescataremos la cultura de cada lugar.

\section{FUENTES Y BIBLIOGRAFÍA CITADAS}

BALLARINI, O. Aprendiz de mago. Mendoza: Ediciones del Autor, 2009.

BAUZÁ, H. F. El mito del héroe; morfología y semántica de la figura heroica. Buenos Aires: FCE, 1998. Disponible en:

https://docs.google.com/document/d/1SCpAQ3SF2HLw4lQ3kuNBgSXrF_hPdu x3y2zjHQzaXkO/edit. Acceso en: 04/10/2021

\section{BUCKINGHAM, D. La infancia materialista. Crecer en la cultura consumista.} Madrid: Ediciones Morata, 2013.

BUFANO, A. Charango. En: VIDELA DE RIVERO, G. (Ed.). Poesías Completas (t. III). Buenos Aires: Ediciones Culturales Argentinas, 1983.

DOLINA, A. La aventura del conocimiento y el aprendizaje. 2011. Disponible en: https://www.academia.edu/37055930/La_Aventura_del_ConocimientoAlejandro_Dolina.pdf. Acceso en: 04/10/2021

KILBEY, E. Niños Desconectados. Cómo pueden crecer nuestros hijos sanos y felices en la era digital. Ediciones EDAF, 2018. Disponible en:

https://books.google.com.ar/books?id=5C9WDWAAQBAJ\&pg=PT10\&lpg=PT1 $0 \& d q=E l i z a b e t h+K i l b e y+q u e+c u b r e+e l+p e r \% C 3 \% A D o d o+q u e+v a+d e+l o s+4+$ $a+$ los $+11+a \% C 3 \% B 1$ os $+m \% C 3 \% A 1 s+o+m e n o s,+e s+u n a+d e+l a s+e t a p a s+m \% C$ $3 \% \mathrm{~A} 1 \mathrm{~s}+$ descuidadas \& source=bl\&ots=LaeCRuy $1 \mathrm{EP} \&$ sig=ACfU3U0DFEoxpqQe7 UCXMmC7RJ03_FTY7A\&hl=es-

419\&sa=X\&ved=2ahUKEwibx_HpmbLzAhWJHLkGHQF5COIQ6AF6BAgJEAM\#v= onepage \&q=Elizabeth\%20Kilbey\%20que\%20cubre\%20el\%20per\%C3\%ADodo\% 20que\%20va\%20de\%20los\%204\%20a\%20los\%2011\%20a\%C3\%B los\%20m\%C3\%A 1s\%20o\%20menos\%2C\%20es\%20una\%20de\%20las\%20etapas\%20m\%C3\%A 1s\% 20descuidadas\&f=false. Acceso en: 04/10/2021 
L'ECUYER, C. Educar en el asombro. $14^{\mathrm{a}}$ ed. Barcelona: Plataforma Actual, 2015.

L'ECUYER, C. Educar en la realidad. $21^{a}$ ed. Barcelona: Plataforma Actual, 2017.

MANES, F. ¿Cómo afectan las nuevas tecnologías a nuestro cerebro? El País. 2015. Disponible en:

https://elpais.com/elpais/2015/12/21/ciencia/1450693458_718084.html. Acceso en: 04/10/2021

MARTíNEZ ARDILA, M. "Cap. II. Referentes teóricos", en El consumo cultural infantil como mediador en la construcción de identidades de niños y niñas: El caso de estudiantes del Ciclo I del Colegio Fanny Mikey I.E.D. Bogotá D. C.: Universidad Pedagógica Nacional. Maestría en Educación, 2016, p. 36-53.

SOSA, A. U. Antología del Meñique. Mendoza: Ediciones El Pelícano, 1963, p. 13. Disponible en:

http://bpd.sanluis.gov.ar:8383/greenstone3/sites/localsite/collect/literat1/ind ex/assoc/HASHOle6.dir/doc.pdf. Acceso en: 04/10/2021

VILLAR, P. Doña Nube y Don Nubarrón. Bogotá, Colombia: Enlace Editoral, 2016. Colección el Tren Dorado.

Recebida em: 12 de setembro de 2021. Aprovada em: 20 de dezembro de 2021.

Publicada em: 07 de janeiro de 2022. 